\title{
On the general form of FIR MIMO biorthogonal partners
}

\author{
Bojan Vrcelj \\ Dept. of Electrical Engineering \\ California Institute of Technology \\ Pasadena, CA 91125
}

\author{
P. P. Vaidyanathan \\ Dept. of Electrical Engineering \\ California Institute of Technology \\ Pasadena, CA 91125
}

\begin{abstract}
The concept of biorthogonal partners has recently been shown to provide a unifying link for various applications in digital signal processing. In particular, the multiple input multiple output (MIMO) case has been associated with several different contexts such as multiwavelet theory and MIMO channel equalization, especially with fractionally spaced equalizers. In most of these applications, the construction of FIR solutions is of high practical importance. In this paper we derive the most general form of FIR MIMO biorthogonal partners. These findings will be tested on a MIMO channel equalization example. ${ }^{1}$
\end{abstract}

\section{Introduction}

The concept of biorthogonal partners has been introduced recently by the authors in both the scalar [5] and the vector case [7, 6]. A pair of digital filters $H(z)$ and $F(z)$ are called biorthogonal partners of each other with respect to an integer $M$ if their cascade $H(z) F(z)$ obeys the Nyquist $(M)$ property. Multiple input multiple output (MIMO) biorthogonal partners are defined using a similar approach [7]. They arise in many standard applications in digital signal processing. Some of them include multiwavelet theory [8], where the prefiltering problem can be treated from the MIMO biorthogonal partner perspective [6]. They are also used in zero-forcing MIMO channel fractionally spaced equalizers (FSEs) [7, 6].

In most of these applications it is of interest to construct FIR MIMO biorthogonal partners. Moreover, if such FIR solutions exist, it is desirable to parameterize them and thus make the search for the most suitable solution (depending on the application) analytically tractable. Some results dealing with this problem were presented in [7] for the case of square matrices and in [6] for the more general, rectangular matrix case. In this paper we further these results by

\footnotetext{
${ }^{1}$ Work supported in part by the ONR grant N00014-99-11002, USA.
}

taking a systematic approach for finding the general set of FIR MIMO biorthogonal partners for an arbitrary decimation ratio $M$. We show that the solution for $M=2$ given in [6] without any generality claims, indeed assumes the general form and that a similar form holds for any $M$. We also provide insights into what can affect the performance of this solution.

We begin by reviewing some properties of MIMO biorthogonal partners including the conditions for the existence of FIR solutions. Next, we derive the general form of FIR MIMO biorthogonal partners and show how they can be optimized in the equalization setting to combat the channel noise. We evaluate the performance of the proposed FIR MIMO equalization method in the section with experimental results.

\subsection{Notations}

If not stated otherwise, all notations are as in [4]. We use the symbol $\downarrow M$ in a box to denote the decimation operation on vectors [turns $\mathbf{x}(n)$ into $\mathbf{x}(M n)$ ]. The expanded version of $\mathbf{x}(n)$

$$
\begin{cases}\mathbf{x}(n / M) & \text { for } n=\operatorname{mul} \text { of } M \\ \mathbf{0} & \text { otherwise }\end{cases}
$$

is similarly obtained as a result of the expander operation which is denoted by the symbol $\uparrow M$. The decimated and expanded versions of $\mathbf{x}(n)$ are denoted by $\mathbf{x}[(n)]_{\downarrow M}$ and $[\mathbf{x}(n)]_{\uparrow M}$. Superscripts $T$ and $\dagger$ operating on vectors or matrices denote the transpose and the transpose conjugate, respectively.

\section{MIMO biothogonal partners}

In the vector case the biorthogonal partner relation is not symmetric, so we distinguish between a left biorthogonal partner (LBP) and a right biorthogonal partner (RBP) [7]. Consider the system in Fig. 1; if it is equal to identity, i.e. if $[\mathbf{H}(z) \mathbf{F}(z)]_{\downarrow M}=\mathbf{I}$, then $\mathbf{H}(z)$ is said to be a left biorthogonal partner of $\mathbf{F}(z)$ with respect to an integer $M$. At the same time we say that $\mathbf{F}(z)$ is a right biorthogonal partner of $\mathbf{H}(z)$ with respect to $M$. Notice that $\mathbf{F}(z)$ and $\mathbf{H}(z)$ can be rectangular matrices. As explained in [7], all the results 


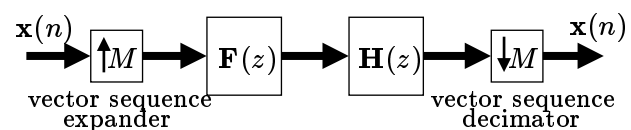

Figure 1: Definition of a biorthogonal partner relation.

that hold for LBPs hold (in a similar form) for RBPs as well, so we consider only LBPs in the following.

Many properties of MIMO biorthogonal partners are derived in [6]. In what follows we are mostly concerned with the case when $\mathbf{F}(z)$ is a polynomial matrix. Under these circumstances, the question is when will there exist a polynomial matrix $\mathbf{H}(z)$ such that $[\mathbf{H}(z) \mathbf{F}(z)]_{\downarrow M}=\mathbf{I}$ ? In other words, when does there exist an FIR LBP of an FIR matrix transfer function? The answer to this question is also provided in [6] and here we just briefly summarize the result.

Let $\mathbf{F}(z)$ be a $p \times r$ polynomial matrix with the Type-2 polyphase form [4]

$$
\mathbf{F}(z)=\sum_{k=0}^{M-1} z^{k} \mathbf{F}_{k}\left(z^{M}\right) .
$$

Then, there exists a polynomial LBP of $\mathbf{F}(z)$ with respect to $M$, namely a $r \times p$ matrix $\mathbf{H}(z)$ if and only if the greatest right common divisor (grcd) [1] of the polyphase components $\left\{\mathbf{F}_{k}(z)\right\}, 0 \leq k \leq M-1$ is a unimodular matrix [1]. (For the purpose of this paper we use an extended definition of grcd's that accommodates rectangular matrices [6].) More generally, it can be shown [6] that under the above conditions, there exist polynomial matrices $\left\{\mathbf{H}_{k}(z)\right\}, 0 \leq k \leq M-1$, such that

$$
\sum_{k=0}^{M-1} \mathbf{H}_{k}(z) \mathbf{F}_{k}(z)=\mathbf{I} .
$$

These polynomial matrices can then be taken as the Type-1 polyphase components of our FIR LBP $\mathbf{H}(z)$. In other words, $\mathbf{H}(z)$ is constructed as

$$
\mathbf{H}(z)=\sum_{k=0}^{M-1} z^{-k} \mathbf{H}_{k}\left(z^{M}\right) .
$$

It is insightful here to note the way matrices $\left\{\mathbf{H}_{k}(z)\right\}$ were originally constructed in $[6]$ for $M=2$. From the construction of a grcd [1] it follows that there exists a unimodular matrix $\mathbf{W}(z)$ such that

$$
r p-r \underbrace{\left[\begin{array}{cc}
p & p \\
\mathbf{W}_{00}(z) & \mathbf{W}_{01}(z) \\
\mathbf{W}_{10}(z) & \mathbf{W}_{11}(z)
\end{array}\right]}_{\mathbf{W}(z)}\left[\begin{array}{c}
\mathbf{F}_{0}(z) \\
\mathbf{F}_{1}(z)
\end{array}\right] p=\left[\begin{array}{c}
\mathbf{P}^{r}(z) \\
\mathbf{0}
\end{array}\right] r
$$

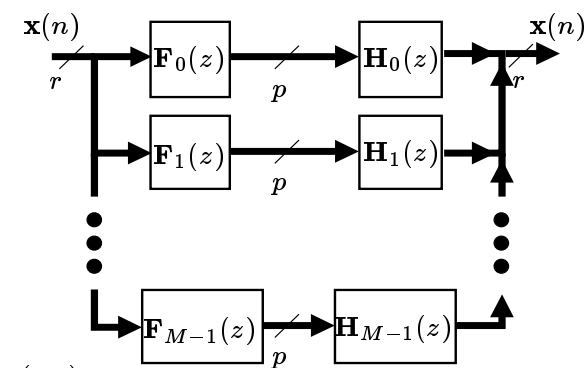

( a )

( b )

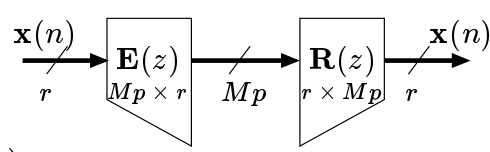

Figure 2: (a)-(b) Construction of general FIR LBPs.

with indicated sizes of the building blocks. Matrix $\mathbf{P}(z)$ appearing on the right hand side of (4) is a grcd of $\mathbf{F}_{0}(z)$ and $\mathbf{F}_{1}(z)$. From the conditions for existence of an FIR LBP we know that $\mathbf{P}(z)$ is unimodular. The polyphase components $\mathbf{H}_{0}(z), \mathbf{H}_{1}(z)$ of valid FIR LBPs are then given by

$\mathbf{H}_{k}(z)=\mathbf{P}^{-1}(z) \mathbf{W}_{0 k}(z)+\mathbf{Q}(z) \mathbf{W}_{1 k}(z)$, for $k=0,1$

where $\mathbf{Q}(z)$ is an arbitrary $r \times(2 p-r)$ polynomial matrix incorporating the degrees of freedom in the construction. In the next section we take a more systematic approach leading to the general form of FIR LBPs.

\section{General form of FIR LBPs}

In view of (2) we can redraw the structure from Fig. 1 as shown in Fig. 2(a). Next, we define the $M p \times r$ and $r \times M p$ polynomial matrices $\mathbf{E}(z)$ and $\mathbf{R}(z)$ as

$$
\begin{aligned}
& \mathbf{E}(z) \triangleq\left[\begin{array}{llll}
\mathbf{F}_{0}^{T}(z) & \mathbf{F}_{1}^{T}(z) & \cdots & \mathbf{F}_{M-1}^{T}(z)
\end{array}\right]^{T} \\
& \mathbf{R}(z) \triangleq\left[\begin{array}{llll}
\mathbf{H}_{0}(z) & \mathbf{H}_{1}(z) & \cdots & \mathbf{H}_{M-1}(z)
\end{array}\right]
\end{aligned}
$$

Then we can redraw Fig. 2(a) as shown in Fig. 2(b). We conclude that constructing an FIR LBP is absolutely equivalent to finding a left polynomial inverse of $\mathbf{E}(z)$, namely $\mathbf{R}(z)$. This is possible as long as $M p \geq r$ and the greatest common divisor (gcd) of all the $M \times M$ minors of $\mathbf{E}(z)$ is a delay [9]. From the previous discussion we conclude that this condition is equivalent to $\operatorname{grcd}\left[\mathbf{F}_{0}(z), \mathbf{F}_{1}(z), \cdots, \mathbf{F}_{M-1}(z)\right]$ being a unimodular matrix.

The Smith form [1] of $\mathbf{E}(z)$ is given by

$$
\mathbf{E}(z)=\mathbf{U}(z) \boldsymbol{\Gamma}(z) \mathbf{V}(z) .
$$


Here $\mathbf{U}(z)$ and $\mathbf{V}(z)$ are $M p \times M p$ and $r \times r$ unimodular matrices respectively and $\boldsymbol{\Gamma}(z)$ is a $M p \times r$ diagonal matrix. Under the above conditions, the elements on its diagonal are nonzero constants or delays, but without loss of generality we can assume that they are all constants. In other words, $\boldsymbol{\Gamma}(z)=\left[\begin{array}{ll}\boldsymbol{\Gamma} & 0\end{array}\right]^{T}$, where $\boldsymbol{\Gamma}$ is a $r \times r$ constant diagonal matrix. Now from (7) we have that the most general form of an FIR left inverse of $\mathbf{E}(z)$ is given by

$$
\mathbf{R}(z)=\mathbf{V}^{-1}(z)\left[\begin{array}{ll}
\boldsymbol{\Gamma}^{-1} & \mathbf{A}(z)
\end{array}\right] \mathbf{U}^{-1}(z),
$$

where $\mathbf{A}(z)$ is any $r \times(M p-r)$ polynomial matrix. It is important to note here that although (8) represents the general form of the solution for FIR LBPs, it still does not provide the complete parameterization of all valid solutions. The reason for this is the fact that the unimodular matrices $\mathbf{U}(z)$ and $\mathbf{V}(z)$ in $(7)$ are not unique; moreover their complete parameterization is still an open problem. In the section with experimental results we demonstrate how changing $\mathbf{U}(z)$ and $\mathbf{V}(z)$ can affect the performance of the FIR LBP.

Given $\mathbf{R}(z)$, the desired polyphase components of an FIR LBP $\mathbf{H}(z)$ are easily obtained using (6). To see how this solution compares to the previously available solution (5), we define the polynomial matrices $\mathbf{D}_{0}(z)$, $\mathbf{D}_{1}(z)$ and $\mathbf{G}(z)$ as

$[\underbrace{\mathbf{D}_{0}^{T}(z)}_{r} \underbrace{\mathbf{D}_{1}^{T}(z)}_{M p-r}]^{T}=\mathbf{D}(z) \triangleq \mathbf{U}^{-1}(z), \quad \mathbf{G}(z) \triangleq \mathbf{V}^{-1}(z)$.

Now, we can rewrite (8) as

$$
\begin{aligned}
& {\left[\begin{array}{llll}
\mathbf{H}_{0}(z) & \mathbf{H}_{1}(z) & \cdots & \mathbf{H}_{M-1}(z)
\end{array}\right]=} \\
& \mathbf{G}(z) \boldsymbol{\Gamma}^{-1} \mathbf{D}_{0}(z)+\underbrace{\mathbf{G}(z) \mathbf{A}(z)}_{\triangleq \mathbf{B}(z)} \mathbf{D}_{1}(z) .
\end{aligned}
$$

Comparing (10) for $M=2$ to (5), we see that if $\mathbf{W}(z)=\mathbf{D}(z)$ and $\mathbf{P}(z)=\mathbf{\Gamma} \mathbf{V}(z)$ these two solutions have exactly the same form, with the free parameter matrix $\mathbf{Q}(z)$ being replaced by $\mathbf{B}(z)$ in (10). Note that $\mathbf{B}(z)$ has the same dimensions as $\mathbf{Q}(z)$ and is also an arbitrary polynomial matrix (this follows from the fact that $\mathbf{G}(z)$ is unimodular).

Since we posed no limitations in the process of deriving the FIR LBP, we conclude that (10) represents the most general form of the solution; therefore, the solution for $M=2$ proposed in [6] is of the general form as well. However, as opposed to [6], here we see that the same equation (10) provides the general form for $M>2$ as well. Therefore, we can use the same optimization procedure for finding the best matrix $\mathbf{A}(z)$ regardless of $M$. This will next be demonstrated in the case when FIR LBPs are used as MIMO channel equalizers.

\section{LBPs as channel equalizers}

The discrete-time equivalent of a MIMO digital communication system with symbol-spaced equalizer (SSE) is shown in Fig. 3(a). The equalizer $\mathbf{H}_{2}(z)$ works at the symbol rate $1 / T$. The discrete versions of the pulse shaping filter and the channel, $\mathbf{G}_{2}(z)$ and $\mathbf{C}_{2}(z)$ are obtained by sampling the corresponding continuous-time impulse responses also at the rate $1 / T$. An ideal equalizer (or a zero-forcing equalizer) $\mathbf{H}_{2}(z)$ is found as a left inverse of the equivalent channel $\mathbf{F}_{2}(z)$.

Several problems with this method have been pointed out in [3]. The receiver is very sensitive to the phase shift at the sampling device; also, sampling at the symbol rate may create some aliasing problems. As for the MIMO transfer function $\mathbf{F}_{2}(z)$, it does not have a left inverse if it is a fat matrix and even if it is not fat, its invertibility will depend on the rank. Furthermore, if $\mathbf{F}_{2}(z)$ is invertible, its inverse is most probably IIR, which often amplifies the noise at the receiver. For all these reasons, a popular alternative is to use a so called fractionally spaced equalizer (FSE) [3]. It is realized by sampling the received waveform at $M$ times the symbol rate, and feeding such oversampled signal to the equalizer, which now operates at the rate $M / T$. In discrete-time this is modeled as shown in Fig. 3(b). The discrete transfer functions $\mathbf{G}(z)$ and $\mathbf{C}(z)$ are obtained after sampling the corresponding continuous-time impulse responses at the rate $M / T$. Thus, the equivalent channel $\mathbf{F}(z)$ in this case is such that $\mathbf{F}_{2}(z)=[\mathbf{F}(z)]_{\downarrow M}$. The simplified scheme is shown in Fig. 4(a). We see that the zeroforcing $\operatorname{FSE~} \mathbf{H}(z)$ is nothing but an LBP of $\mathbf{F}(z)$ with respect to $M$. Our goal is to find an FIR ZFE $\mathbf{H}(z)$ that will not severely amplify the channel noise. This will be achieved by using the flexibility in the design of FIR LBPs as described in Sec. 3; we apply a technique similar to that employed in [6].

From Sec. 3 we recall that Fig. 4(a) can be redrawn as in Fig. 4(b) when both $\mathbf{F}(z)$ and $\mathbf{H}(z)$ are FIR. The noise vector process needs to get modified as well. The noise from Fig. 4(a) is assorted into polyphase components and they are distributed over the $M$ vector channels in Fig. 4(b). We know that our equalizer $\mathbf{H}(z)$ (rather its polyphase components) needs to be of the form (10), and to obtain the best solution we consider the equivalent noise model shown in Fig. 5(a) and try to minimize the power of the output noise process $\hat{\mathbf{e}}(n)$. 


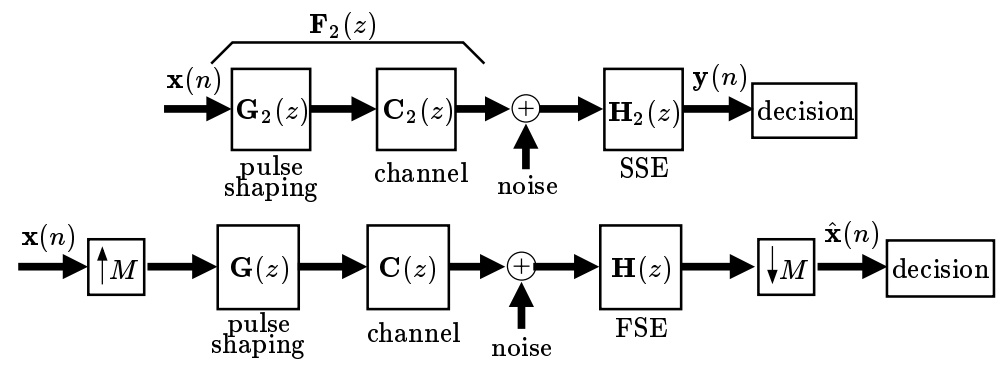

Figure 3: (a) Discrete-time equivalent of a digital communication system with SSE; the equivalent channel is $\mathbf{F}_{2}(z)=\mathbf{C}_{2}(z) \mathbf{G}_{2}(z)$. (b) Digital communication system equalized with FSE $\mathbf{H}(z)$.

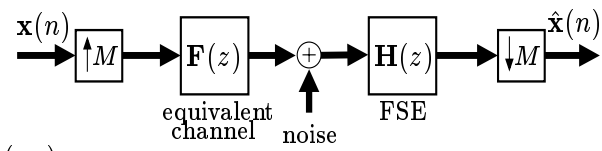

( a )

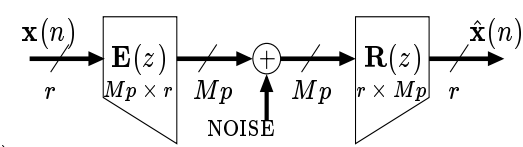

Figure 4: (a)-(b) MIMO FSEs and MIMO LBPs.

First, recall that the FIR matrix inverse $\mathbf{R}(z)$ can be written as in (8), with $\mathbf{U}(z), \mathbf{V}(z)$ and $\boldsymbol{\Gamma}$ defined in (7). Thus we can redraw Fig. 5(a) as in Fig. 5(b). Furthermore, $\mathbf{R}(z)$ is equivalently given by (10) and therefore the equivalent noise model can be shown as in Fig. 5(c). The $r \times(M p-r)$ polynomial matrix of free parameters $\mathbf{A}(z)$ is now replaced by another $r \times(M p-r)$ polynomial matrix of free parameters $\mathbf{B}(z)$ and our goal is to find the optimal $\mathbf{B}(z)$ of a given order $N_{B}-1$ that will minimize the noise power. From Fig. 5(c) we see that the optimal $\mathbf{B}(z)$ is nothing but a matrix Wiener filter [2] for recovering the desired vector signal $-\mathbf{u}(n)$ given the vector process $\mathbf{v}(n)$. Let $\mathbf{C}(z) \triangleq \mathbf{V}^{-1}(z) \boldsymbol{\Gamma}^{-1} \mathbf{D}_{0}(z)$ and let the matrices $\mathbf{B}_{i}, \mathbf{C}_{i}$ and $\mathbf{D}_{i}$ represent the impulse responses of $\mathbf{B}(z), \mathbf{C}(z)$ and $\mathbf{D}_{1}(z)$ respectively. Next define the $r \times N_{C} M p$ matrix $\mathcal{C}$ and the $(M p-r) N_{B} \times M p\left(N_{B}+N_{D}-1\right)$ matrix $\mathcal{D}_{1}$ as

$$
\begin{aligned}
& \mathcal{C} \triangleq\left[\begin{array}{llllll}
\mathbf{C}_{0} & \mathbf{C}_{1} & \ldots & \mathbf{C}_{N_{C}-1}
\end{array}\right] \\
& \mathcal{D}_{1} \triangleq\left[\begin{array}{cccccc}
\mathbf{D}_{0} & \ldots & \mathbf{D}_{N_{D}-1} & \mathbf{0} & \ldots & \mathbf{0} \\
\mathbf{0} & \mathbf{D}_{0} & \ldots & \mathbf{D}_{N_{D}-1} & \ldots & \mathbf{0} \\
\vdots & & \ddots & & \ddots & \\
\mathbf{0} & \ldots & \mathbf{0} & \mathbf{D}_{0} & \ldots & \mathbf{D}_{N_{D}-1}
\end{array}\right]
\end{aligned}
$$

We also define the $N_{B}(M p-r) \times 1$ vector process $\mathcal{V}(n)$
( a )

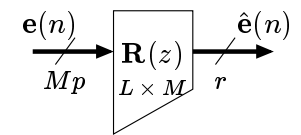

( b )

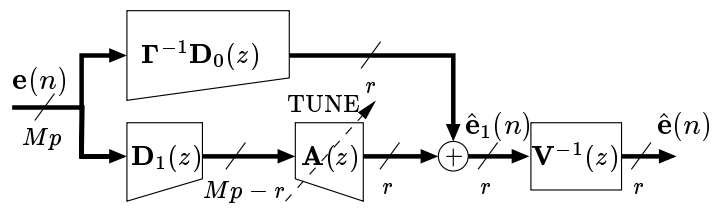

( c )

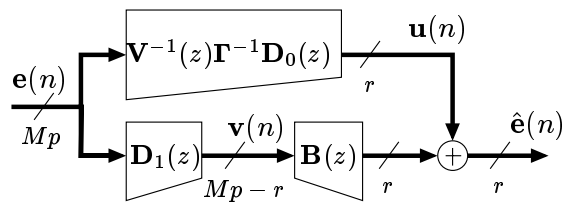

Figure 5: (a)-(c) Finding the optimal FIR LBP.

and the $r \times N_{B}(M p-r)$ matrix $\mathcal{B}$ as

$$
\begin{aligned}
\mathcal{V}(n) & \triangleq\left[\begin{array}{llll}
\mathbf{v}^{T}(n) & \mathbf{v}^{T}(n-1) & \cdots & \mathbf{v}^{T}\left(n-N_{B}+1\right)
\end{array}\right]^{T} \\
\mathcal{B} & \triangleq\left[\begin{array}{llll}
\mathbf{B}_{0} & \mathbf{B}_{1} & \cdots & \mathbf{B}_{N_{B}-1}
\end{array}\right] .
\end{aligned}
$$

By the orthogonality principle we have that $E\left\{[\mathcal{B V}(n)+\mathbf{u}(n)] \mathcal{V}^{\dagger}(n)\right\}=\mathbf{0}(E\{\cdot\}$ denotes the expectation), which provides the optimal $\mathcal{B}$ as

$$
\mathcal{B}=-E\left\{\mathbf{u}(n) \mathcal{V}^{\dagger}(n)\right\} \cdot \mathcal{R}_{\mathcal{V} \mathcal{V}}^{-1}
$$

where $\mathcal{R}_{\mathcal{V} \mathcal{V}}$ is the autocorrelation matrix of $\mathcal{V}(n)$. Given the definitions (11) and referring to Fig. 5(c) we see that $\mathbf{u}(n)=\mathcal{C} \mathcal{E}_{N_{C}}$ and $\mathcal{V}(n)=\mathcal{D}_{1} \mathcal{E}_{N_{D}+N_{B}-1}$, where $\mathcal{E}_{N}$ denotes the $N M p \times 1$ vector of concatenated input noise vectors $\mathbf{e}(n-i), 0 \leq i \leq N-1$. Substituting in (13) we get the optimal $\mathbf{B}(z)$ to be given by its impulse response matrix

$$
\mathcal{B}=-\mathcal{C R}_{\epsilon}\left(1: N_{C} L,:\right) \mathcal{D}_{1}^{\dagger}\left(\mathcal{D}_{1} \mathcal{R}_{\epsilon}\left(1: N_{D}, 1: N_{D}\right) \mathcal{D}_{1}^{\dagger}\right)^{-1}
$$



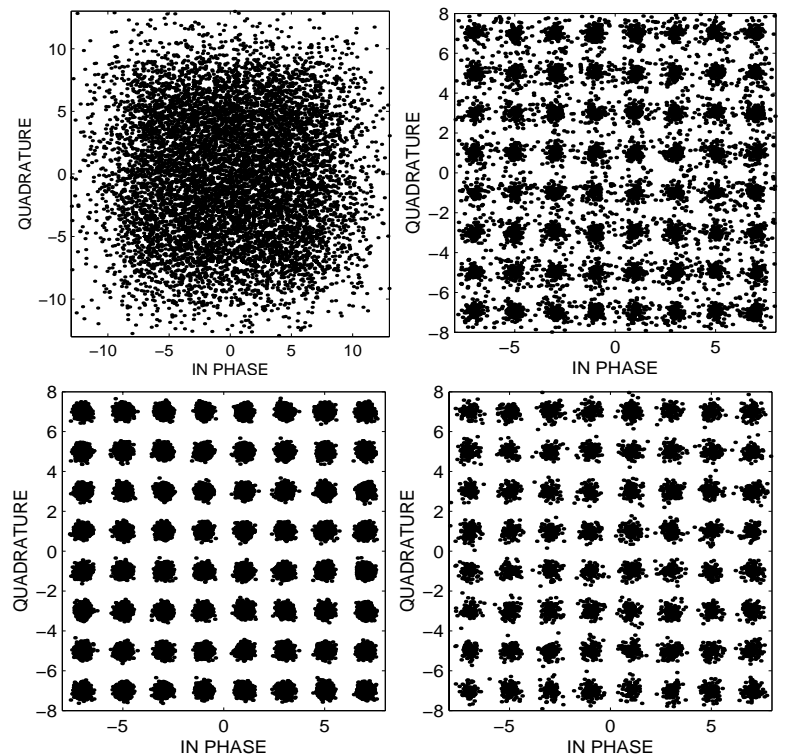

Figure 6: Equalization results. Clockwise, starting from upper left: SSE, plain FIR FSE, optimized FIR FSE as in [6] and optimized FIR FSE as in Sec. 4.

Here $\mathcal{R}_{\epsilon}$ is a $L\left(N_{B}+N_{D}-1\right) \times L\left(N_{B}+N_{D}-1\right)$ autocorrelation matrix of the input noise process, and we use Matlab's notation $\mathbf{X}(1: N,:)$ to denote the matrix made of the first $N$ rows of $\mathbf{X}$.

\section{Experimental results}

In the experiment we compare the equalization results of a $3 \times 1$ input vector sequence $\mathbf{x}(n)$ whose scalar components were iid coming from a $64-\mathrm{QAM}$ constellation. Four different methods were used:

1. traditional IIR SSE [Fig. 3(a)],

2. plain FIR FSE obtained as an LBP with redundancy set to zero $[\mathbf{A}(z)=\mathbf{0}$ in (8)],

3. optimized FIR FSE according to $[7,6]$ [see (5)] with $\mathbf{Q}(z)$ of order three, and

4. optimized FIR FSE as in Sec. 4 with $\mathbf{B}(z)$ of order three obtained as in (14).

The corresponding scattering diagrams are shown in Fig. 6, with the probabilities of error (clockwise in Fig. 6) $0.24,0.0036,5.33 \times 10^{-5}$ and $5.26 \times 10^{-6}$. The equivalent channel $\mathbf{F}(z)$ in Fig. $4($ a) was a $3 \times 3$ polynomial matrix of order three and can be found at [10]. The oversampling ratio $M=2$. The noise was white and the SNR at the channel output was 28 $\mathrm{dB}$. Matrix $\mathbf{W}(z)$ in (4) was obtained using the grcd construction algorithm from [1]. As mentioned in Sec. 3 , matrices $\mathbf{U}(z)$ and $\mathbf{V}(z)$ in $(7)$ are not unique and this is exploited in the last example. Notice that there is a factor of 10 improvement in the probability of error with respect to the method from [6] and this can mostly be attributed to the choice of $\mathbf{U}(z)$ and $\mathbf{V}(z)$. All the three unimodular matrices used $[\mathbf{W}(z), \mathbf{U}(z)$ and $\mathbf{V}(z)$ ] can be found at [10].

\section{Concluding remarks}

In this paper we consider the problem of finding the general form of FIR MIMO biorthogonal partners. We show that the previously proposed solutions can also be reduced to this general form. However, the form derived here is valid for arbitrary decimation ratios $M$ and provides some further insights. The results are tested on MIMO channel equalization examples where it is shown that different choices of the Smith form decomposition result in different performances.

\section{References}

[1] T. Kailath, Linear Systems. Prentice Hall, Inc., Englewood Cliffs, N.J., 1980.

[2] C. W. Therrien, Discrete Random Signals and Statistical Signal Processing. Prentice-Hall, Englewood Cliffs, NJ, 1992.

[3] J. R. Treichler, I. Fijalkow and C. R. Johnson, Jr., "Fractionally spaced equalizers: how long should they really be?," IEEE Signal Processing Magazine, pp. 65-81, May 1996.

[4] P. P. Vaidyanathan, Multirate Systems and Filter Banks. Prentice-Hall, Englewood Cliffs, NJ, 1995.

[5] P. P. Vaidyanathan and B. Vrcelj, "Biorthogonal partners and applications," IEEE Trans. Signal Processing, vol. 49(5), pp. 1013-1027, May 2001.

[6] B. Vrcelj and P. P. Vaidyanathan, "MIMO biorthogonal partners and applications," IEEE Trans. Signal Proc, to appear.

[7] B. Vrcelj and P. P. Vaidyanathan, "Theory of MIMO biorthogonal partners and their application in channel equalization," Proceedings ICC, Helsinki, Finland, June 2001.

[8] X.-G. Xia, J. S. Geronimo, D. P. Hardin and B. W. Suter, "Design of prefilters for discrete multiwavelet transforms," IEEE Trans. Signal Processing, vol. 44, pp. 25-35, Jan. 1996.

[9] X.-G. Xia, "New precoding for intersymbol interference cancellation using nonmaximally decimated multirate filterbanks with ideal FIR equalizers," IEEE Trans. Signal Processing, vol. 45(10), pp. 2431-2441, Oct. 1997.

[10] http://www.systems.caltech.edu/bojan/papers/asilo2001.html 